Research in Astron. Astrophys.

http://www.raa-journal.org http://www.iop.org/journals/raa

\title{
B3 0749+460A: A New "Changing-Look" Active Galactic Nucleus Associated with X-ray Spectral Slope Variations
}

\author{
J. Wang ${ }^{1,2}$, W. K. Zheng ${ }^{3}$, D. W. Xu ${ }^{2,4}$, T. G. Brink ${ }^{3}$, A. V. Filippenko ${ }^{3,5}$, C. Gao ${ }^{1,2,4}$, S. S. \\ Sun $^{1,2,4}$ and J. Y. Wei ${ }^{2,4}$ \\ ${ }^{1}$ Guangxi Key Laboratory for Relativistic Astrophysics, School of Physical Science and Technology, \\ Guangxi University, Nanning 530004, China; wj@ nao.cas.cn \\ 2 Key Laboratory of Space Astronomy and Technology, National Astronomical Observatories, \\ Chinese Academy of Sciences, Beijing 100101, China; dwxu@ nao.cas.cn \\ 3 Department of Astronomy, University of California, Berkeley, CA 94720-3411, USA \\ 4 School of Astronomy and Space Science, University of Chinese Academy of Sciences, Beijing, \\ People's Republic of China \\ ${ }^{5}$ Miller Institute for Basic Research in Science, University of California, Berkeley, CA 94720, USA
}

\begin{abstract}
Here we report an identification of B3 0749+460A as a new double-peaked local "changing-look" active galactic nucleus (CL-AGN) in terms of our multi-epoch spectroscopic analysis. By comparing our new spectra taken in 2021 with the ones taken by the Sloan Digital Sky Survey in 2004, BOSS in 2013, and MaNGA in 2016, we reveal type transitions of Seyfert (Sy) $1.9 \rightarrow$ Sy1.8 $\rightarrow$ Sy 1.9. In the transitions, the classical broad $\mathrm{H} \alpha$ emission fades away since 2013, and disappears in our 2021 spectrum, although the absence of broad $\mathrm{H} \beta$ can be traced back to at least 2016. A follow-up observation in $\mathrm{X}$-rays by the Swift/XRT reveals that (1) the X-ray emission level gradually decreases since 2005; and (2) the X-ray spectrum is soft in the optical "turn-off" state and hard in the "turn-on" state. We argue that the disappearance of the classical broad $\mathrm{H} \alpha$ emission can be likely explained by the disk-wind broad-line-region model, in which the CL phenomenon is sensitive to luminosity in individual AGNs.
\end{abstract}

Key words: galaxies: Seyfert — galaxies: nuclei $-\mathrm{X}$-rays: galaxies — quasars: emission lines — quasars: individual (B3 0749+460A)

\section{INTRODUCTION}

Depending on the existence of broad Balmer emission lines (full width at half-maximum intensity (FWHM) $\gtrsim 2000 \mathrm{~km} \mathrm{~s}^{-1}$ ) in their optical spectra, active galactic nuclei (AGNs) can be classified into Type 1 and Type 2 . The latter lack broad Balmer emission lines in their spectra, widely understood by the unified model (e.g., Antonucci 1993) in which its central engine is obscured by the dusty torus along the line of sight to an observer. This orientation-based model has, however, recently been challenged by the rarely discovered "changing-look" (CL) phenomenon, in which some AGNs show a spectral transition between Type 1, intermediate, and Type 2 within a timescale of years to decades (e.g., MacLeod et al. 2010, 2016; Shapovalova et al. 2010; Shappee et al. 2014; LaMassa et al. 2015; McElroy et al. 2016; Ruan et al. 2016; Runnoe et al. 2016; Parker et al. 2016; Gezari et al. 2017; Sheng et al. 2017, 2020; Stern et al. 2018; Yang et al. 2018; Wang et al. 2018b, 2019, 2020a; Frederick et al. 2019; Trakhtenbrot et al. 2019; Yan et al. 2019; Guo et al. 2019; Ai et al. 2020; Graham et al. 2020; Kollatschny et al. 2018, 
2020). The widely accepted standard disk model has also been challenged by the CL phenomenon in terms of the viscosity crisis (e.g., Lawrence 2018, and references therein).

The physical origin of CL-AGNs is still an open question, although the scenario involving clumpy obscuration can be almost entirely excluded by light echos in the mid-infrared (e.g., Sheng et al. 2017) and by spectropolarimetry (e.g., Hutsemekers et al. 2019). There is, in fact, some evidence supporting the idea that the CL phenomenon results from a variation of the accretion power of a supermassive black hole (SMBH, e.g., H. Feng et al. 2021), even though the underlying physical connection is still poorly understood. On the one hand, some previous studies argue that CL-AGNs tend to be biased toward the low Eddington ratio end $\left(L / L_{\mathrm{Edd}} ; L_{\mathrm{Edd}}=1.26 \times 10^{38} M_{\mathrm{BH}} / M_{\odot} \mathrm{erg} \mathrm{s}^{-1}\right.$ is the Eddington luminosity; e.g., MacLeod et al. 2019; Wang et al. 2020b), which is roughly consistent with the expectation of the disk-wind broad-line region (BLR) model (e.g., Elitzur \& Ho 2009; Nicastro 2000; Elitzur \& Shlosman 2006).

On the other hand, Ruan et al. (2019) and Ai et al. (2020, and references therein) argue that the CL phenomenon might be related to an accretion-state transition similar to that occurring in X-ray binaries (XRBs), after taking into account a "V"-shaped correlation between $\mathrm{X}$-ray hardness and $L / L_{\text {Edd }}$ identified in a few CL-AGNs. This conclusion, however, is argued against by Wang et al. (2020b), who found that the CL phenomenon identified in UGC 3223 is related to its X-ray emission level, rather than to its $\mathrm{X}$-ray hardness ratio.

At the current stage, understanding of the CL phenomenon is greatly hampered by the scarcity of identified cases; there are only $\sim 100$ CL-AGNs identified by multi-epoch spectroscopy. Among these, there are just 8 objects showing repeat type transitions (e.g., Parker et al. 2019; Wang et al. 2020a; Marin et al. 2019, and references therein), and only a couple of cases with a comparison study in X-rays.

Hon et al. (2020) recently reported a significant line-profile variation in the local AGN B3 0749+460A. In this paper, we identify the object as a new double-peaked CL-AGN thanks to our elaborate spectral analysis, and we report a follow-up observation in X-rays, which enables us to claim an X-ray spectral slope-dependent CL phenomenon. The paper is organized as follows. Section 2 presents our optical spectroscopic and X-ray observations, along with the data reduction. The spectral analysis in both optical and X-rays is described in Section 3. Sections 4 and 5 present the results and discussion, respectively. A significant line-profile variation in this object was reported by Hon et al. (2020), who argue that it is an unusual object; we include a comparison with this study. A $\Lambda$ CDM cosmological model with parameters $\mathrm{H}_{0}=70 \mathrm{~km} \mathrm{~s}^{-1} \mathrm{Mpc}^{-1}, \Omega_{\mathrm{m}}=0.3$, and $\Omega_{\Lambda}=0.7$ is adopted throughout.

\section{OBSERVATIONS AND DATA REDUCTION}

B3 0749+460A (=NPM1G+46.0092, $\left.\alpha=07^{\mathrm{h}} 52^{\mathrm{m}} 44^{\mathrm{s}} .2, \delta=+45^{\circ} 56^{\prime} 58^{\prime \prime} ; \mathrm{J} 2000\right)$ is a local AGN at a redshift of $z=0.0518$. The object was classified as a Seyfert 1.9 galaxy in the catalog of quasars and active nuclei (12th ed.; Veron-Cetty \& Veron 2006) and the NASA/IPAC Extragalactic Database (NED). It is part of a sample of CL-AGN candidates we selected by cross-matching the local partially obscured AGNs extracted from the Sloan Digital Sky Survey (SDSS) DR7 with the Wide-field Infrared Survey Explorer (WISE) catalog (Wright et al. 2010).

\subsection{Optical Spectroscopy}

\subsubsection{Observations}

We performed long-slit spectroscopic observations with the $2.16 \mathrm{~m}$ telescope (Fan et al. 2016) at the Xinglong Observatory of the National Astronomical Observatories, Chinese Academy of Sciences (NAOC) on 2021 January 12 (UT dates are used throughout this paper), and with the $3 \mathrm{~m}$ Shane telescope at Lick Observatory on 2021 January 21 . The first spectrum was taken with the Beijing Faint Object Spectrograph and Camera (BFOSC) that is equipped with a back-illuminated E2V55-30 AIMO CCD. We used a long slit of width $2^{\prime \prime}$ oriented in the north-south direction. Our spectral resolution was

\footnotetext{
1 http://ned.ipac.caltech.edu/.
} 
$\sim 10 \AA$ and the wavelength coverage was $3850-8200 \AA$. The exposure time was $2 \times 2400$ s. Wavelength calibration was carried out with spectra of iron-argon comparison lamps. In order to minimize the effects of atmospheric dispersion (Filippenko 1982), the spectrum was obtained as close to the meridian as possible.

The second spectrum was obtained with the Kast double spectrograph (Miller \& Stone 1993) mounted on the $3 \mathrm{~m}$ Shane telescope. Grism 600/4310 was used on the blue side and grating 300/7500 on the red side, providing respective resolutions of $\sim 5 \AA$ and $\sim 12 \AA$ and a range of $3600-10,700 \AA$. The long $2^{\prime \prime}$ slit was aligned near the parallactic angle (Filippenko 1982) to minimize differential light losses caused by atmospheric dispersion, and the exposure time was $1500 \mathrm{~s}$.

Flux calibration of both spectra was carried out with observations of Kitt Peak National Observatory standard stars (Massey et al. 1988).

\subsubsection{Data Reduction}

One-dimensional (1D) spectra were extracted from the raw images by using the IRAF package and standard procedures, including bias subtraction and flat-field correction. Both extracted 1D spectra were then calibrated in wavelength and in flux with the corresponding comparison lamp and standard stars. The accuracy of the wavelength calibration is better than $1 \AA$ for the Kast spectrum and better than $2 \AA$ for the BFOSC spectrum. The telluric A-band (7600-7630 $\AA$ ) and B-band (around $6860 \AA$ ) absorption produced by atmospheric $\mathrm{O}_{2}$ molecules were removed from both spectra by using the observation of the corresponding standard star.

Each calibrated spectrum was then corrected for the Galactic extinction of $A_{V}=0.226 \mathrm{mag}$ (Schlafly \& Finkbeiner 2011) taken from the NED. The correction was applied by assuming the $R_{V}=3.1$ extinction law of our Galaxy (Cardelli et al. 1989). Both spectra were then transformed to the rest frame. The two resulting spectra are displayed in Figure 1 (lower two curves).

\subsection{X-ray Follow-up Observation}

We proposed X-ray and ultraviolet (UV) follow-up observations of the object at the beginning of 2021 by using the Neil Gehrels Swift Observatory (Gehrels et al. 2004) X-ray telescope (XRT) and Ultraviolet/Optical Telescope (UVOT). The object was targeted $(\mathrm{ObsID}=00037357002)$ by XRT and UVOT simultaneously on 2021 February 12. The exposure times were $1400 \mathrm{~s}$ in XRT Photon Counting (PC) mode and $1400 \mathrm{~s}$ for the UVOT. The UVOT image is, however, useless because of heavy contamination caused by the bright star in the field of view.

The XRT data taken in the PC mode were reduced with HEASOFT version 6.27.2, along with the corresponding CALDB version 20190910. The source spectrum was then extracted from the image in a circular region with a radius of $20.0^{\prime \prime}$. An adjacent region free of any sources was adopted to extract the background-sky spectrum. The corresponding ancillary response file was produced by the task xrtmkarf. The total XRT count rate in the $0.3-10 \mathrm{keV}$ range was estimated to be $(4.62 \pm 0.44) \times 10^{-2}$ count s$^{-1}$.

\section{ANALYSIS AND RESULTS}

In order to identify the CL phenomenon in B3 $0749+460 \mathrm{~A}$ and to reveal the underlying physics, spectral analysis was performed on the optical and X-ray spectra described in Section 2.

\subsection{Optical Spectroscopy: A CL-AGN}

Figure 1 compares the new spectra taken in 2021 with the previous spectra, which were obtained in different epochs, extracted from the SDSS DR7, BOSS, and Mapping Nearby Galaxies at Apache Point Observatory (MaNGA) survey. The MaNGA spectrum is extracted from the datacube by an aperture

\footnotetext{
${ }^{2}$ IRAF is distributed by NOAO, which is operated by AURA, Inc., under cooperative agreement with the U.S. National Science Foundation (NSF).
} 


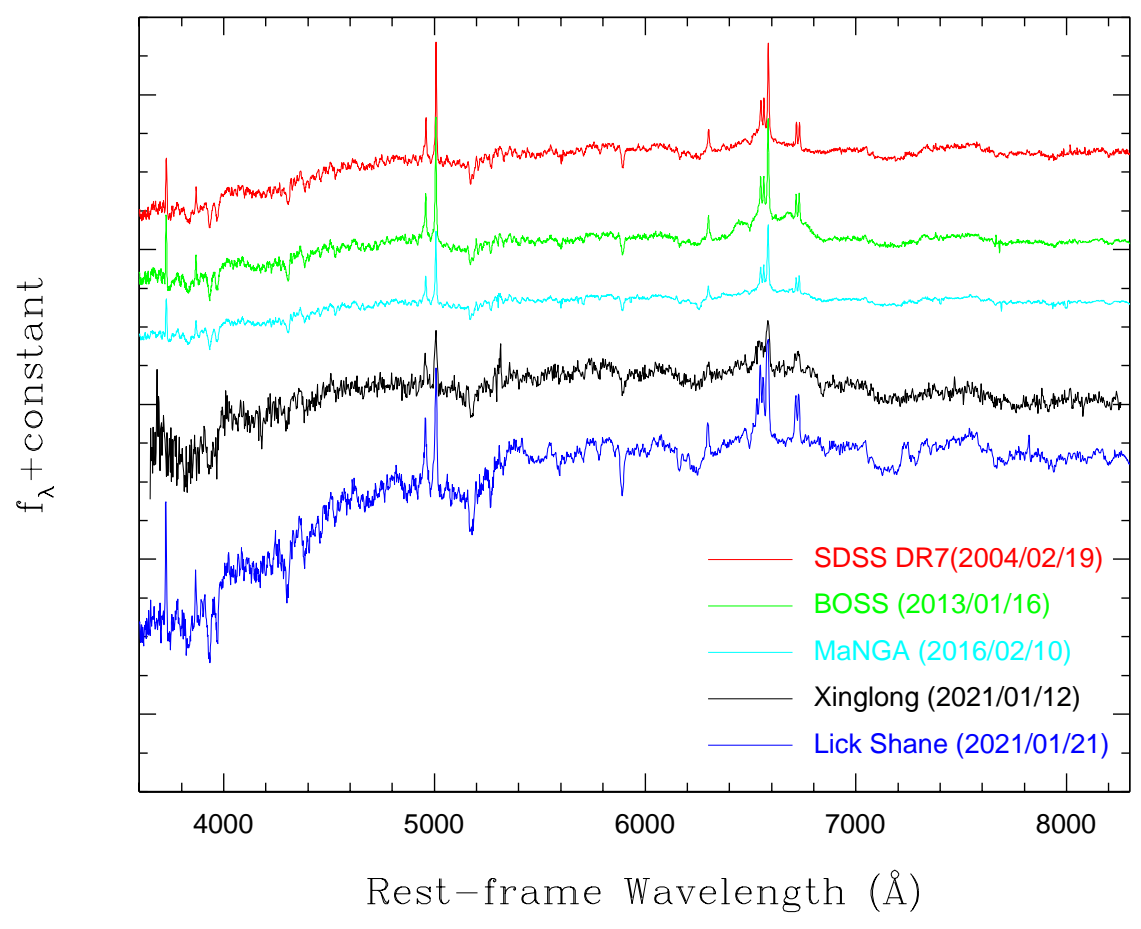

Fig. 1 A comparison between the rest-frame spectra of B3 0749+460A taken at different epochs. The two new spectra obtained with the Xinglong $2.16 \mathrm{~m}$ telescope and the Shane $3 \mathrm{~m}$ telescope are displayed by the lower two curves. The three spectra extracted from the SDSS DR7, BOSS, and MaNGA spectral database are shown by the upper three curves. The spectra are vertically shifted by an arbitrary amount for clarity.

with a diameter of $3^{\prime \prime}$. At first glance, one can see clearly a significant variation of the $\mathrm{H} \alpha$ line profile. The SDSS spectrum taken in 2013 shows the existence of a very broad, double-peaked $\mathrm{H} \alpha$ component that is, however, absent or weak in the other four spectra. The following spectral analysis allows us to identify the object as a local repeat CL-AGN.

\subsubsection{Subtraction of the Starlight Component}

We first remove the starlight component from each of the five spectra by modeling the stellar features with a linear combination of the first seven eigenspectra through $\chi^{2}$ minimization, where the eigenspectra are built from the standard single stellar population spectral library developed by Bruzual \& Charlot (2003). The minimization is carried out over the rest-frame wavelength range from 3700 to $8000 \AA$, except for the regions with strong emission lines - Balmer lines (both narrow and broad components), [O III] $\lambda \lambda 4959,5007,[\mathrm{~N} \mathrm{II}] \lambda \lambda 6548,6583$, [S II] $\lambda \lambda 6716,6731$, [O II] $\lambda 3727$, [O III] $\lambda 4363$, and [O I] $\lambda 6300$. Intrinsic extinction due to the host galaxy described by the Galactic extinction curve with $R_{V}=3.1$ is also involved in the modeling. A potential featureless continuum contributed by the central 


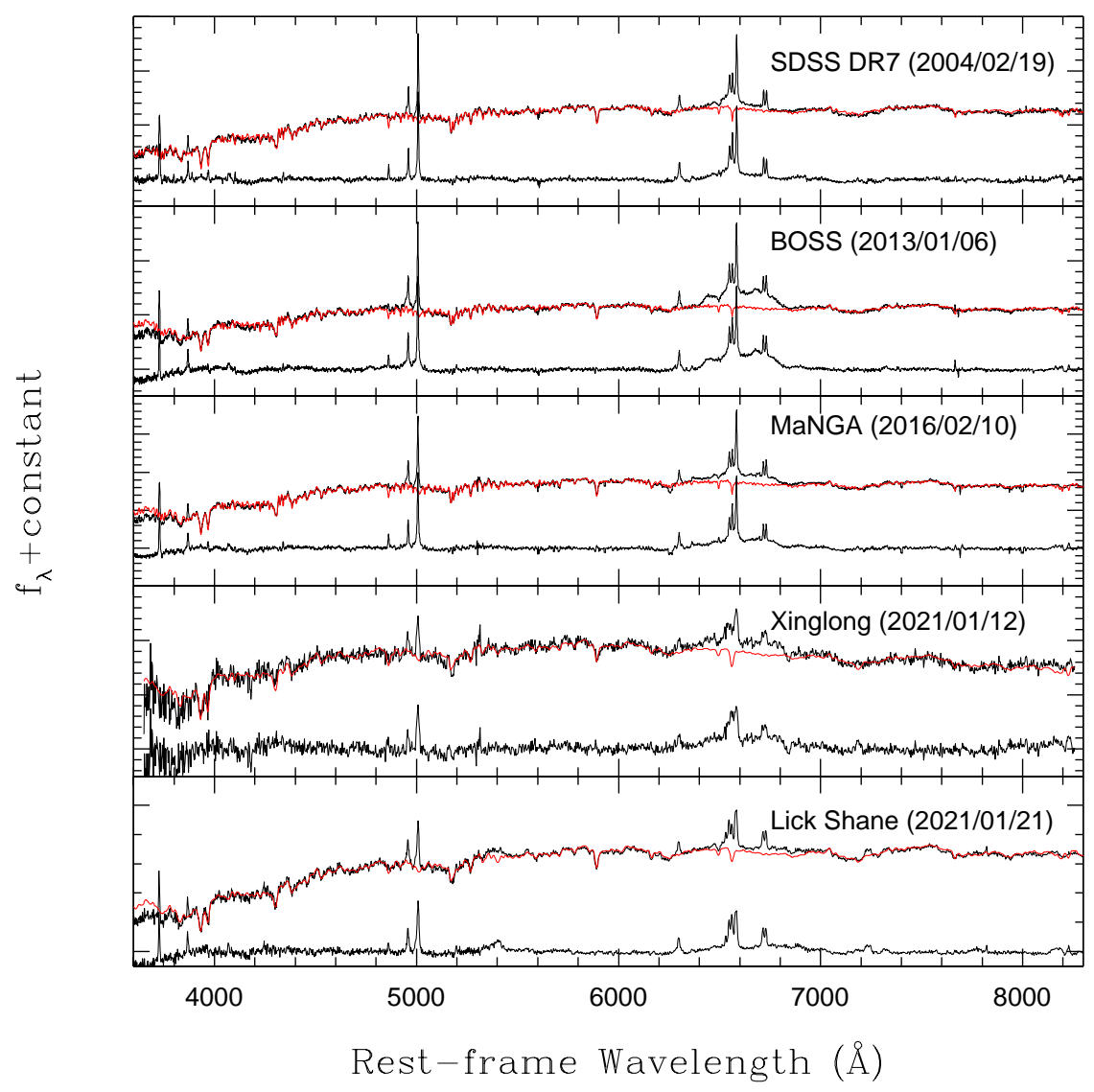

Fig. 2 An illustration of the modeling and removal of the stellar continuum for the five spectra (see the main text for the details). In each panel, the top black curve shows the observed rest-frame spectrum, overplotted by the best-fit continuum indicated by the red curve. The black curve underneath shows the continuum-removed emission-line spectrum.

AGN is ignored in the minimization; it is almost degenerate with the spectrum of early-type stars, we do not focus on the stellar population in this study, and the effect caused by the featureless continuum is usually slight (e.g., Wang 2015). The starlight templates are convolved with a fixed Gaussian profile in advance before the minimization for the Xinglong spectrum, because the observed absorption features are dominated by the instrumental profile. The subtraction of the starlight component is illustrated in Figure 2.

\subsubsection{Line-Profile Modeling}

After removing the starlight component, we model the emission-line profiles in each of the spectra by a linear combination of a set of Gaussian profiles in the $\mathrm{H} \alpha$ and $\mathrm{H} \beta$ regions by the SPECFIT task (Kriss 1994 ) in IRAF. The 2021 Xinglong spectrum is not included in the profile modeling simply because the nearly contemporaneous 2021 Shane spectrum has better spectral resolution and higher signal-tonoise ratio (S/N). In the modeling, the line-flux ratios of the [O III] $\lambda \lambda 4959,5007$ and the [N II] $\lambda \lambda 6548$, 6583 doublets are fixed to their theoretical values of 1:3. A sum of a narrow component and a blueshifted 
broad component is required to reproduce the [O III] $\lambda \lambda 4959,5007$ line profiles in all three spectra (e.g., Boroson 2005; Zhang et al. 2013; Harrison et al. 2014; Woo et al. 2017; Wang et al. 2011, 2018a).

The Balmer-line models are described as follows.

- 2004 SDSS spectrum. The $\mathrm{H} \alpha$ line profile can be well reproduced by a combination of narrow $\left(\mathrm{FWHM} \approx 400 \mathrm{~km} \mathrm{~s}^{-1}\right)$ and broad $\left(\mathrm{FWHM} \approx 7800 \mathrm{~km} \mathrm{~s}^{-1}\right)$ component 3 . A broad component is not needed to reproduce the profile of $\mathrm{H} \beta$.

- 2013 BOSS spectrum. In addition to a classical broad component with FWHM $\approx 3000 \mathrm{~km} \mathrm{~s}^{-1}$, a double-peaked broad H $\alpha$ (e.g., Halpern, \& Filippenko 1988; Eracleous \& Halpern 1994; Strateva et al. 2003; Popovic et al. 2003) line containing redshifted and blueshifted broad components is necessary to properly reproduce the observed $\mathrm{H} \alpha$ profile; thus, a total of three broad components is required. Double-peaked Balmer emission lines are expected to originate from the rotating gas in an accretion disk (e.g., Chen et al. 1989; Popovic et al. 2004; Bon et al. 2009a,b; Storchi-Bergmann et al. 2017).

Initially, we model the $\mathrm{H} \beta$ profile by a sum of a narrow and a broad $\mathrm{H} \beta$ emission component; however, this results in a residual on the blue side of [O III] $\lambda 4959$ line. Since the intrinsic profile of the [O III] $\lambda 4959$ line must be physically identical to that of the [O III] $\lambda 5007$ line, the residual motivates us to reproduce the emission-line spectrum in the $\mathrm{H} \beta$ region by an additional broad redshifted $\mathrm{H} \beta$ emission component.

We argue that both $\mathrm{H} \alpha$ and $\mathrm{H} \beta$ show the same double-peaked broad-line profiles in the 2013 SDSS spectrum, because of the comparable bulk velocities of the broad redshifted components. The bulk velocities are calculated to be $5980 \pm 90 \mathrm{~km} \mathrm{~s}^{-1}$ and $5310 \pm 140 \mathrm{~km} \mathrm{~s}^{-1}$ for $\mathrm{H} \alpha$ and $\mathrm{H} \beta$, respectively. The broad blueshifted $\mathrm{H} \beta$ is likely undetectable owing to its weakness.

- 2016 MaNGA spectrum. Except for its significant weakness, the modeling of the $\mathrm{H} \alpha$ line profile is similar to that of the 2013 BOSS spectrum. The $\mathrm{H} \beta$ emission line can, however, be modeled well by a single narrow component.

- 2021 Shane spectrum. The classical $\mathrm{H} \alpha$ broad component with a width of $\sim 10^{3} \mathrm{~km} \mathrm{~s}^{-1}$ is not necessary for reproducing the line profile. The $\mathrm{H} \alpha$ broad emission line can be described by a fainter double-peaked profile, compared to the MaNGA spectrum. Again, a single narrow Gaussian can reproduce the $\mathrm{H} \beta$ line profile properly.

The line-profile models are displayed in Figure 3 for the $\mathrm{H} \beta$ (left) and $\mathrm{H} \alpha$ (right) regions, and the results are listed in Table 1. All of the reported errors correspond to the $1 \sigma$ significance level and include only the uncertainties caused by the fitting, not the removal of the stellar continuum.

To summarize, in addition to a significant variation of the broad $\mathrm{H} \alpha$ line profile, the spectral modeling and analysis enable us to reveal weak, broad (and most likely double-peaked) $\mathrm{H} \beta$ components in the SDSS 2013 spectrum, but not in the other three spectra, suggesting that the object is a CL-AGN with type transitions of Sy1.9 $\rightarrow$ Sy1.8 $\rightarrow$ Sy 1.9 (Type 1.8 and 1.9 AGNs are classified according the existence and absence of a weak broad $\mathrm{H} \beta$ line, respectively; e.g., Osterbrock \& Ferland 2006.), although the prototypical CL refers to a transition between Type 1 and Type 2 spectra.

\subsubsection{Estimation of Black Hole Mass and Eddington Ratio}

After the line-profile modeling, we estimate the black hole mass $\left(M_{\mathrm{BH}}\right)$ and Eddington ratio $\left(L / L_{\mathrm{Edd}}\right)$ in terms of the modeled $\mathrm{H} \alpha$ broad emission line through the traditional method described by Wang et al. (2020a). Briefly, $M_{\mathrm{BH}}$ can be estimated by the calibration (Greene \& Ho 2007)

$$
M_{\mathrm{BH}}=3.0 \times 10^{6}\left(\frac{L_{\mathrm{H} \alpha}}{10^{42} \mathrm{erg} \mathrm{s}^{-1}}\right)^{0.45}\left(\frac{\mathrm{FWHM}_{\mathrm{H} \alpha}}{1000 \mathrm{~km} \mathrm{~s}^{-1}}\right)^{2} M_{\odot}
$$

\footnotetext{
${ }^{3}$ All line widths are not corrected for the instrumental spectral resolution throughout of the paper.
} 


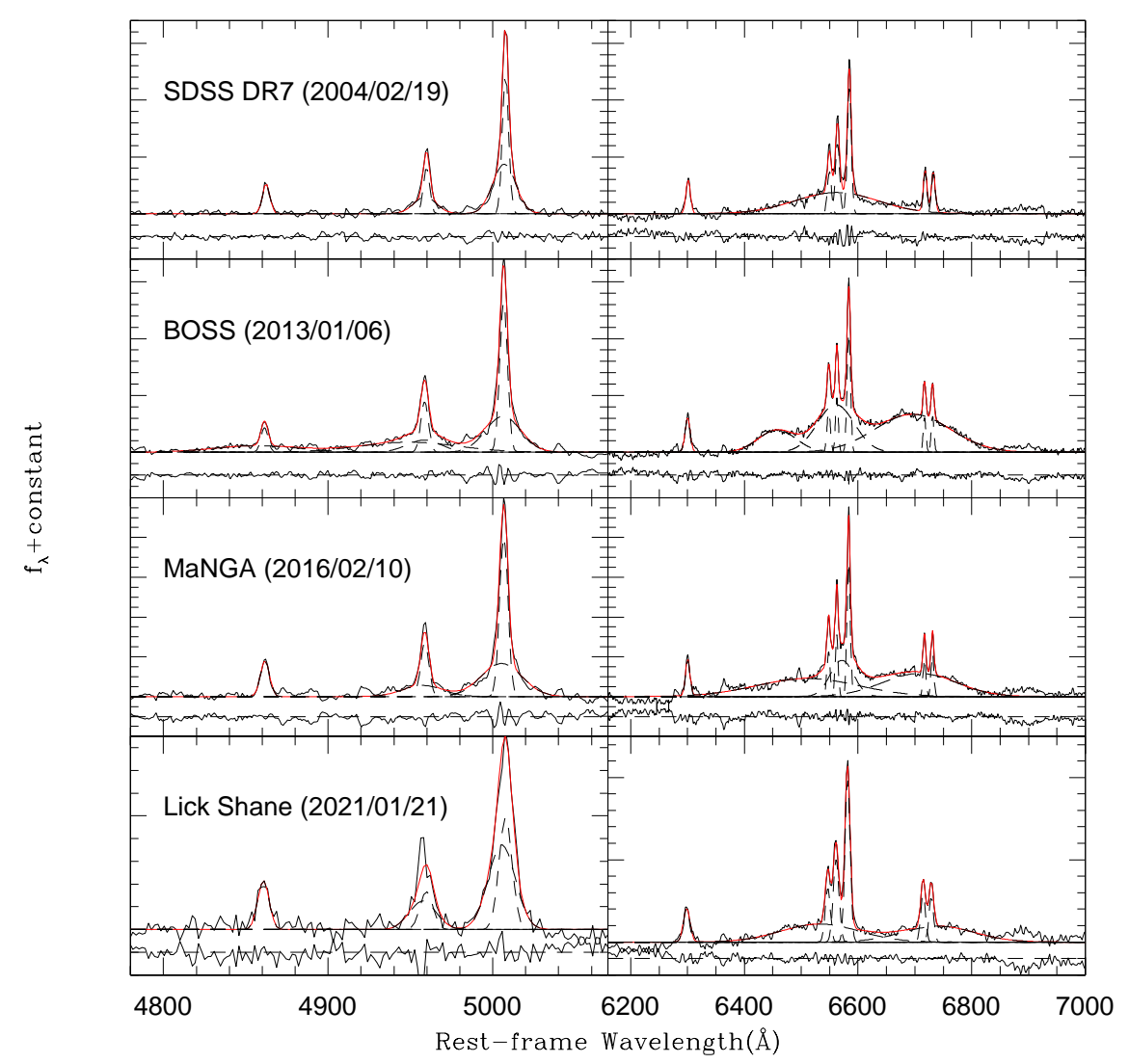

Fig. 3 An illustration of the line-profile modeling with a linear combination of a set of Gaussian functions for $\mathrm{H} \beta$ (left panels) and $\mathrm{H} \alpha$ (right panels). In each panel, the modeled stellar continuum has already been removed from the original observed spectrum. The observed and modeled line profiles are plotted with the black and red solid lines, respectively. Each Gaussian function is shown by a dashed line. The subpanel underneath the line spectrum presents the residuals between the observed and modeled profiles.

and $L / L_{\mathrm{Edd}}$ through a bolometric correction of $L_{\mathrm{bol}}=9 \lambda L_{\lambda}(5100 \AA$ ) (e.g., Kaspi et al. 2000), where (Greene \& Ho 2005)

$$
\lambda L_{\lambda}(5100 \AA)=2.4 \times 10^{43}\left(\frac{L_{\mathrm{H} \alpha}}{10^{42} \operatorname{erg~s}^{-1}}\right)^{0.86} \operatorname{erg~s}^{-1} .
$$

The 2004 SDSS spectrum is used as a reference in the subsequent comparison. In deriving the broad $\mathrm{H} \alpha$ luminosity $L_{\mathrm{H} \alpha}$, the measured broad $\mathrm{H} \alpha$ line fluxes in the BOSS, MaNGA, and Shane spectra are first scaled by a factor determined by equaling the total [O III] $\lambda 5007$ line fluxes to that of the reference one (e.g., Peterson et al. 2000). Then, the intrinsic extinction is corrected from the narrow-line flux ratio $\mathrm{H} \alpha / \mathrm{H} \beta$ by assuming the Balmer decrement of standard Case $\mathrm{B}$ recombination and a Galactic extinction curve with $R_{V}=3.1$. The value of $M_{\mathrm{BH}}$ is estimated to be $\sim 1.3 \times 10^{8} M_{\odot}$ based on the 2004 SDSS spectrum, which we use as a fiducal value because of the regular and symmetric $\mathrm{H} \alpha$ emission-line profile. With this value of $M_{\mathrm{BH}}$, the lowest row in Table 1 reports the estimated $L / L_{\mathrm{Edd}}$, where $L_{\mathrm{bol}}$ is estimated from the total $\mathrm{H} \alpha$ broad emission. One can see a relation between $L / L_{\mathrm{Edd}}$ and spectral types. 
Table 1 Results of Line-Profile Modeling and Analysis

\begin{tabular}{|c|c|c|c|c|}
\hline $\begin{array}{l}\text { Parameters } \\
\text { (1) }\end{array}$ & $\begin{array}{c}2004 \text { Feb. } 19 \\
(2)\end{array}$ & $\begin{array}{c}2013 \text { Jan. } 06 \\
\text { (3) }\end{array}$ & $\begin{array}{c}2016 \text { Feb. } 10 \\
\text { (4) }\end{array}$ & $\begin{array}{c}2021 \text { Jan. } 21 \\
(5)\end{array}$ \\
\hline \multicolumn{5}{|c|}{ Line flux $\left(10^{-15} \mathrm{erg} \mathrm{s}^{-1} \mathrm{~cm}^{-2}\right)$} \\
\hline$F([\mathrm{O}$ III $] \lambda 5007)$ & $13.4 \pm 1.0$ & $16.3 \pm 0.4$ & $10.3 \pm 0.3$ & $25.1 \pm 4.1$ \\
\hline$F\left(\mathrm{H} \beta_{\mathrm{b}}\right)$ & ...…… & $3.7 \pm 0.4$ & ...n....... & . \\
\hline$F\left(\mathrm{H} \beta_{\mathrm{b} 2}\right)$ & & $6.6 \pm 0.8$ & & \\
\hline$F\left(\mathrm{H} \alpha_{\mathrm{b}}\right)$ & $34.9 \pm 1.3$ & $34.2 \pm 0.6$ & $9.4 \pm 0.5$ & \\
\hline$F\left(\mathrm{H} \alpha_{\mathrm{b} 1}\right)$ & …..... & $16.4 \pm 0.4$ & $20.4 \pm 0.5$ & $37.8 \pm 1.3$ \\
\hline$F\left(\mathrm{H} \alpha_{\mathrm{b} 2}\right)$ & $\ldots \ldots \ldots \ldots$ & $55.8 \pm 0.5$ & $18.9 \pm 0.3$ & $30.7 \pm 0.9$ \\
\hline \multicolumn{5}{|c|}{ Line width $\left(\mathrm{km} \mathrm{s}^{-1}\right)$} \\
\hline $\mathrm{FWHM}\left(\mathrm{H} \beta_{\mathrm{b}}\right)$ & & $3760 \pm 400$ & & \\
\hline $\mathrm{FWHM}\left(\mathrm{H} \beta_{\mathrm{b} 2}\right)$ & & $4260 \pm 520$ & & \\
\hline $\operatorname{FWHM}\left(\mathrm{H} \alpha_{\mathrm{b}}\right)$ & $7860 \pm 250$ & $3500 \pm 60$ & $2210 \pm 80$ & \\
\hline $\mathrm{FWHM}\left(\mathrm{H} \alpha_{\mathrm{b} 1}\right)$ & $\cdots$ & $3640 \pm 80$ & $9760 \pm 220$ & $7440 \pm 260$ \\
\hline $\operatorname{FWHM}\left(\mathrm{H} \alpha_{\mathrm{b} 2}\right)$ & & $7000 \pm 70$ & $6820 \pm 140$ & $6660 \pm 30$ \\
\hline$L / L_{\mathrm{Edd}}$ & 0.005 & 0.012 & 0.009 & 0.005 \\
\hline
\end{tabular}

\subsection{X-ray Energy Spectrum}

\subsubsection{Swift/XRT Spectra in 2008, 2016, and 2021}

- Swift/XRT 2021 spectrum. Because of its low count rate, we attempt to model the X-ray energy spectrum of B3 0749+460A by XSPEC (v12.11, Arnaud 1996) with two simple models over the 0.3-10 keV range in terms of the C-statistic (Cash 1979; Humphrey et al. 2009; Kaastra 2017). The adopted models can be expressed as wabs $*$ zwabs $*$ powerlaw and wabs $* z$ pcfabs $*$ powerlaw. In both cases, the Galactic hydrogen column density is fixed to be $N_{\mathrm{H}}=5.55 \times 10^{20} \mathrm{~cm}^{-2}$ (Kalberla et al. 2005). Both models return similar results characterized by a quite soft X-ray spectrum with a photon index of $\Gamma=2.8 \pm 0.5$.

- Swift/XRT 2008 spectrum. The object was observed by the Swift/XRT in pointing mode with an exposure time of $9000 \mathrm{~s}$ on 2008 February 24, and is included in the second Swift/XRT Point Source (2SXP) Catalog (1SXPS J075244.4+455655; Evans et al. 2020). The energy spectrum is extracted by the method described in Section 2.2, and analyzed by the same models applied to the 2021 spectrum. Both best-fitted models return a hard X-ray spectrum with a photon index of $\Gamma=1.7 \pm$ 0.1 .

- Chandra 2016 spectrum. The spectrum of the object was taken by ACIS-S onboard the Chandra satellite on 2016 December 21. We reduced the data with software CIAO version 4.13 and the corresponding calibration database CALDB 4.9.5. The extracted spectrum is then again modeled by the XSPEC package, which returns a quite hard power-law spectrum with a photon index of $\Gamma=1.5 \pm 0.1$. In addition to the continuum models aforementioned, two Gaussian functions are required to model the weak $\mathrm{Ar}$ and $\mathrm{Ca} \mathrm{K} \alpha$ emission lines at 3.0 and $3.7 \mathrm{keV}$, respectively.

The best fits and their parameters are shown in Figure 4 and listed in Table 2. In the table, all of the quoted uncertainties correspond to a $90 \%$ significance level.

\subsubsection{Spectral Slope Transition in the CL Phenomenon}

Here we compare the new X-ray observation with the previous ones to reveal the potential physical process occurring in the CL phenomenon of the object. In addition to the 2SXP Catalog, the object is in the XMM-Newton Slew Survey Source Catalog (XMM-SSC, XMMSL2 J075244.7+455654). There are three detections at different epochs in the XMM-SSC catalog from 2005 to 2011.

The comparison is shown in Table 3. Columns (2) and (3) list the intrinsic fluxes in the $0.3-10 \mathrm{keV}$ $(F(0.3-10) \mathrm{keV})$ and the observed flux in the $0.2-12 \mathrm{keV}(F(0.2--12) \mathrm{keV})$ bands, respectively. In order to compare with the previous values, the tabulated fluxes in 2021 are obtained from our fitting 


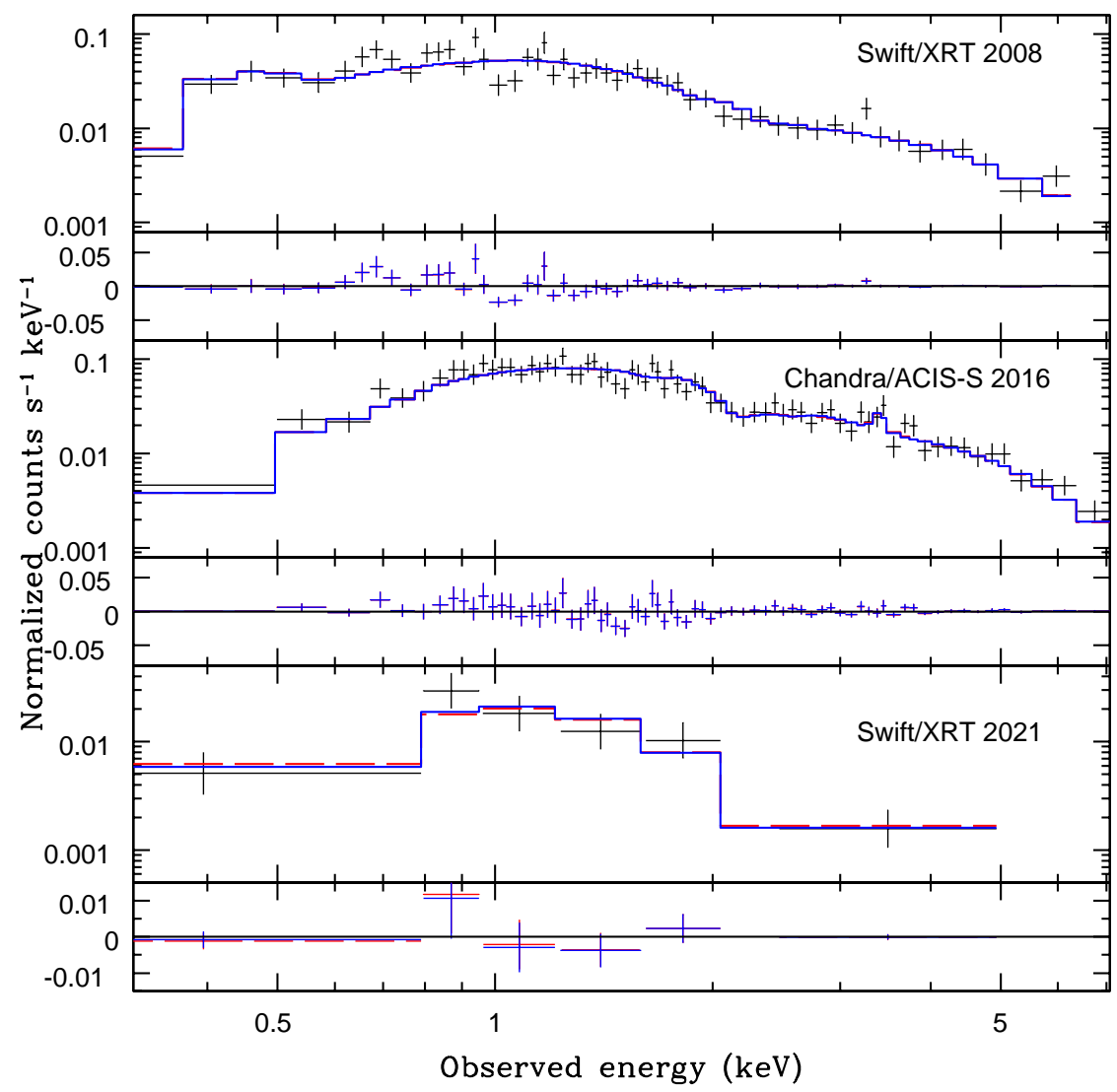

Fig. 4 X-ray spectrum of B3 0749+460A taken at different epochs and the best-fit spectral model expressed as wabs $*$ zwabs $*$ powerlaw (Model 1, red-dashed line) and wabs $*$ zpcfabs $*$ powerlaw (Model 2, blue-solid line). The subpanel underneath the spectrum shows the deviations, in units of counts $\mathrm{s}^{-1} \mathrm{keV}^{-1}$, of the observed data from the best-fit model.

Table 2 X-ray spectra fit parameters of B3 0749+460A.

\begin{tabular}{|c|c|c|c|c|c|}
\hline \multirow[t]{2}{*}{ Parameter } & \multicolumn{3}{|c|}{ Value } & \multirow{2}{*}{$\begin{array}{l}\text { Units } \\
\text { (5) }\end{array}$} & \multirow[t]{2}{*}{ Description } \\
\hline & $\begin{array}{l}2008 \\
(2)\end{array}$ & $\begin{array}{l}2016 \\
(3)\end{array}$ & $\begin{array}{l}2021 \\
(4)\end{array}$ & & \\
\hline \multicolumn{6}{|c|}{ Model 1-wabs * zwabs * powerlaw } \\
\hline$N_{\mathrm{H}}$ & $0.01_{-0.01}^{+0.03}$ & $<0.01$ & $0.25_{-0.09}^{+0.12}$ & $10^{22} \mathrm{~cm}^{-2}$ & Local column density \\
\hline$\Gamma$ & $1.65_{-0.11}^{+0.14}$ & $1.48_{-0.09}^{+0.10}$ & $2.75_{-0.45}^{+0.54}$ & & Power-law index \\
\hline$F(2-10 \mathrm{keV})$ & $2.63_{-0.33}^{+0.36} \times 10^{-12}$ & $1.50_{-0.18}^{+0.17} \times 10^{-12}$ & $3.70_{-0.29}^{+0.45} \times 10^{-13}$ & $\operatorname{erg~s}^{-1} \mathrm{~cm}^{-2}$ & Unabsorbed flux \\
\hline Cash statistics & $53.16 / 50=1.063$ & $54.18 / 66=0.821$ & $2.46 / 4=0.615$ & & \\
\hline \multicolumn{6}{|c|}{ Model 2 - wabs $* z p c f a b s *$ powerlaw } \\
\hline$\eta_{\mathrm{H}}$ & $0.01_{-0.01}^{+1.33}$ & $<0.01$ & $0.30_{+0.64}^{-0.26}$ & $10^{22} \mathrm{~cm}^{-2}$ & Local equivalent column density \\
\hline$f$ & 0.95 & $\ldots \ldots \ldots$ & 0.95 & & Dimensionless covering fraction (fixed) \\
\hline$\Gamma$ & $1.64_{-0.10}^{+0.15}$ & $1.48_{-0.09}^{+0.10}$ & $2.76_{-1.08}^{+1.01}$ & & Power-law index \\
\hline$F(2-10 \mathrm{keV})$ & $2.65_{-0.32}^{+0.36} \times 10^{-12}$ & $1.50_{-0.18}^{+0.17} \times 10^{-12}$ & $3.84_{-0.28}^{+0.27} \times 10^{-13}$ & $\operatorname{erg~s}{ }^{-1} \mathrm{~cm}^{-2}$ & Unabsorbed flux \\
\hline Cash statistics & $56.33 / 49=1.150$ & $54.18 / 66=0.809$ & $2.77 / 3=0.923$ & & \\
\hline
\end{tabular}


Table 3 Comparison of X-ray emission

\begin{tabular}{|c|c|c|c|c|c|c|c|c|}
\hline Year of Obs. & $\begin{array}{l}F(0.3-10) \mathrm{keV} \\
\quad\left(10^{-12} \mathrm{erg} \mathrm{s}\right.\end{array}$ & $\begin{array}{l}F(0.2-12) \mathrm{keV} \\
\left.-1 \mathrm{~cm}^{-2}\right)\end{array}$ & $\overline{\mathrm{HRR} 1_{\mathrm{XMM}}}$ & HR1 Swift $_{\text {Sw }}$ & $\overline{\text { HR2 } 2_{\text {Swift }}}$ & $\overline{\text { Sp. type }}$ & Mission & Reference \\
\hline (1) & $(2)$ & (3) & (4) & (5) & (6) & (7) & (8) & (9) \\
\hline 2005 & .. & $10.3 \pm 1.9$ & $-0.50 \pm 0.18$ & $\cdots$ & - $>3$ & Sy1.9 & XMM-Newton & 1 \\
\hline $2006^{a}$ & & $6.7 \pm 1.8$ & & & & $\ldots \ldots$ & XMM-Newton & 1 \\
\hline 2008 & $5.2 \pm 0.3$ & $\ldots \ldots \ldots \ldots \ldots$ & $\ldots$ & $0.07 \pm 0.04$ & $-0.09 \pm 0.04$ & …... & Swift & 2 \\
\hline & $4.3 \pm 0.3$ & $4.3 \pm 0.4$ & $-0.35 \pm 0.03$ & $0.07 \pm 0.04$ & $-0.08 \pm 0.04$ & & Swift & This work \\
\hline 2011 & & $6.7 \pm 2.3$ & $0.21 \pm 0.35$ & & 年 & Sy1.8 & XMM-Newton & 1 \\
\hline 2016 & $2.2 \pm 0.2$ & $2.3 \pm 0.2$ & $-0.43 \pm 0.03$ & $-0.05 \pm 0.03$ & $-0.12 \pm 0.04$ & Sy1.9 & Chandra & This work \\
\hline 2021 & $2.1_{-0.8}^{+1.0}$ & $0.8_{-0.3}^{+0.6}$ & $-0.41 \pm 0.16$ & $-0.42 \pm 0.14$ & $-0.27 \pm 0.20$ & Sy1.9 & Swift & This work \\
\hline
\end{tabular}

Note 1. References: 1: XMM-SSC (2017); 2: Evans et al. (2020)

${ }^{a}$ There is no reported value of HR1 in the catalog due to the lack of the count rate in the B7 channel.

with the model of wabs * zwabs * powerlaw. The XMM-Newton hardness ratid 4 is listed in Column (4). Columns (5) and (6) are the hardness ratios HR1 and HR2 defined by Evans et al. (2020) for the Swift mission 5 . All of the uncertainties shown in the table correspond to a $90 \%$ significance level, after taking into account proper error propagation. For the 2008 Swift/XRT observation, the hardness ratios obtained in the current work are highly consistent with the values reported in the 2SXP Catalog (Evans et al. 2020).

The upper panel in Figure 5 shows the long-term variation of the X-ray flux and hardness ratio $\mathrm{HR} 1_{\mathrm{XMM}}$ of the object. On the one hand, as shown in the table, the X-ray flux decreases gradually by more than an order of magnitude during the period from 2005 to 2021, which does not follow the spectral-type transition. On the other hand, based on the hardness ratios at different epochs, one can see from the comparison that the CL phenomenon identified in B30749+460A tends to be related to the X-ray slope. The X-ray spectrum is found to be soft (HR1 $=-0.50 \pm 0.18$ in 2005 and HR1 $=-0.41 \pm 0.16$ in 2021) at the "turn-off" state with a Seyfert 1.9-like spectrum, and to be hard $(\mathrm{HR} 1=0.21 \pm 0.35)$ in 2011 when the object was possibly at the "turn-on" state with a Seyfert 1.8-like spectrum.

\section{CONCLUSIONS AND DISCUSSION}

We identify B3 0749+460A as a new local CL-AGN by comparing SDSS spectra previously taken in 2004, 2013, and 2016 with new spectra taken by us in 2021. The object shows type transitions of Sy 1.9 $\rightarrow$ Sy1.8 $\rightarrow$ Sy1.9. In particular, the classical broad H $\alpha$ component fades away from 2013 to 2021. A follow-up observation in X-rays taken by the Swift/XRT enables us to reveal (1) gradually decreasing X-ray emission since 2005, and (2) the X-ray spectrum is soft in the optical "turn-off" state with a Seyfert 1.9-like spectrum. Also, the X-ray spectrum is hard in the "turn-on" state with a Seyfert 1.8-like spectrum, although there is a separation of about two years between the optical and X-ray observations.

\subsection{Broad H $\beta$ Emission}

Here we argue that the identified spectral-type transitions are not caused by the removal of the stellar continuum. The left panel in Figure 6 shows the differential spectra of the object obtained by adopting the 2004 SDSS spectrum as the reference, after scaling the others according to the total [O III] $\lambda 5007$ line flux. One can see that, in addition to the redshifted $\mathrm{H} \alpha$ broad component, there is a bump at the red side of $\mathrm{H} \beta$ in the 2013 BOSS spectrum. Three arguments support our belief that the bump represents the

\footnotetext{
4 The XMM-Newton hardness ratio is defined as $\left(\mathrm{R}_{\mathrm{b} 7}-\mathrm{R}_{\mathrm{b} 6}\right) /\left(\mathrm{R}_{\mathrm{b} 7}+\mathrm{R}_{\mathrm{b} 6}\right)$, where $R_{\mathrm{b} 7}$ and $R_{\mathrm{b} 6}$ are the hard $(2-12 \mathrm{keV})$ and soft $(0.2-2 \mathrm{keV})$ band rates, respectively.

5 The Swift hardness ratios HR1 and HR2 are defined as HR1 $=(M-S) /(M+S)$ and HR2 $=(H-M) /(H+M)$, where $S, M$, and $H$ are the count rates in the $0.3-1 \mathrm{keV}, 1-2 \mathrm{keV}$, and $2-10 \mathrm{keV}$ energy bands, respectively.
} 


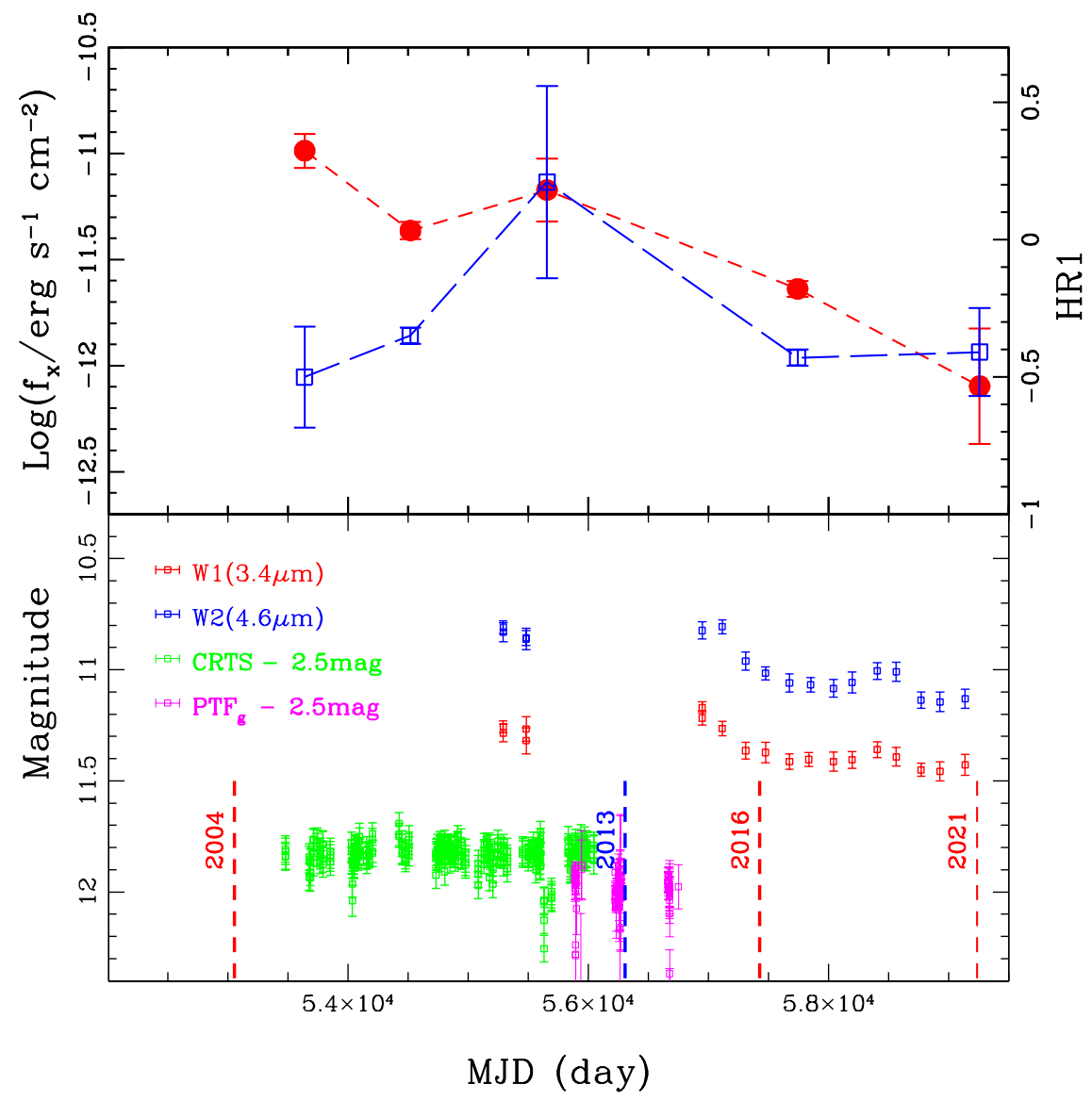

Fig. 5 Upper panel: Evolution of the observed X-ray flux in 0.2-12keV (red symbol) and XMM-Newton HRI (blue symbol). Lower panel: Multi wavelength light curves of B3 0749+460A detected by the Catalina Real-time Transient Survey (Drake et al. 2011), Palomar Transient Factory survey (Law et al. 2009)and WISE. The light curves are binned by averaging the measurements within one day. The vertical dashed lines mark the epochs of optical spectra, where the blue line denotes the "turn-on" state with a Seyfert 1.8-like spectrum having weak broad $\mathrm{H} \beta$ emission lines, and the red ones the "turn-off" state with a Seyfert 1.9-like spectrum. The corresponding years are marked beside the vertical lines.

redshifted $\mathrm{H} \beta$ broad component. First, as shown in the right panel of the figure, the two bumps exhibit quite similar profiles, after taking into account a measured bump flux ratio of 3 that is comparable to the average Balmer ratio of the broad Blamer lines in AGNs (Dong et al. 2006). Second, based on simple Gaussian fitting, the two bumps show comparable bulk redshift velocities with respect to the corresponding rest-frame wavelength. The measured bulk velocities are $\Delta v=5895 \pm 87 \mathrm{~km} \mathrm{~s}^{-1}$ and $5310 \pm 140 \mathrm{~km} \mathrm{~s}^{-1}$ for $\mathrm{H} \alpha$ and $\mathrm{H} \beta$, respectively. Finally, the bump in the $\mathrm{H} \beta$ region is unlikely to result from He I $\lambda 4922$ broad emission (e.g., Veron et al. 2002), simply because the broad He I $\lambda 5876$ emission line is not detected in the observed and differential spectra.

\subsection{A Comparison with Hon et al.}

Although our new spectrum taken in 2021 is comparable to the 2016 MaNGA spectrum shown by Hon et al. (2020), our current study is quite improved in the spectral analysis when compared to Hon 


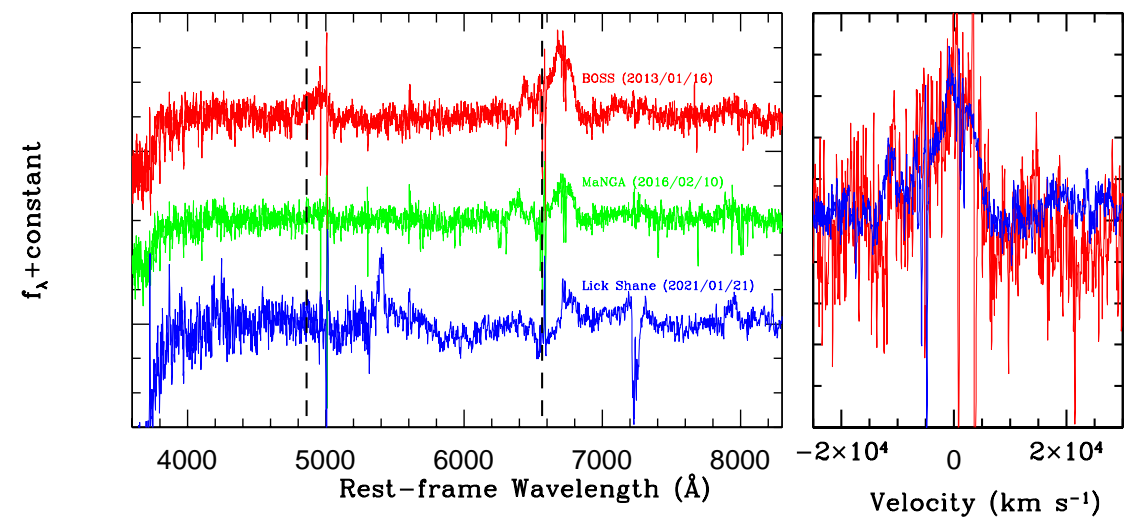

Fig. 6 Left panel: A comparison of the differential spectra taken in 2013, 2016, and 2021, when the SDSS 2004 spectrum is used as a reference. Before the subtraction, the two spectra are scaled in flux level according to their total [O III] $\lambda 5007$ line fluxes. The two vertical lines mark the rest-frame wavelengths of the $\mathrm{H} \beta$ and $\mathrm{H} \alpha$ emission lines. One can see clearly a redshifted broad $\mathrm{H} \beta$ component in the BOSS 2013 spectrum, but not in the 2021 Shane spectrum. In the Shane spectrum, the features around $5400 \AA$ are artificial, caused by uncertainty in the low-S/N region where the blue-side and red-side CCD spectra are combined. Right panel: A comparison of profiles of the two redshifted bumps, after scaling them by a measured flux ratio of the two bumps of 3 (red, $\mathrm{H} \beta$; blue, $\mathrm{H} \alpha$ ).

et al. (2020). The stellar-component subtraction, line-profile modeling, and a comparison within the differential spectrum enable us to argue for the existence of a broad $\mathrm{H} \beta$ line in the 2013 SDSS spectrum, which suggests that a CL phenomenon occurred in the object. Hon et al. (2020) argued against a typical CL phenomenon in the object, because of the short variation timescale of $\sim 1000 \mathrm{~d}$. However, a CL timescale of $\sim 1-3 \mathrm{yr}$ has been revealed in previous investigations (e.g., Merloni et al. 2015; Runnoe et al. 2016; Macleod et al. 2016). In addition, the supernova (SN) explanation of the SDSS 2013 spectrum suggested by Hon et al. (2020) can be potentially excluded by the lack of $\mathrm{Ca}$ II triplet emission in the spectrum. Finally, taking into account the 2016 MaNGA spectrum, the object experienced type transitions of Sy1.9 $\rightarrow$ Sy $1.8 \rightarrow$ Sy1.9 within a duration of $13 \mathrm{yr}$ (from 2004 to 2016), although the broad $\mathrm{H} \alpha$ emission is found to be further weaker in 2021 than in 2016. 


\subsection{Physical Implications}

The lower panel in Figure 5 shows the multiwavelength light curves of B4 0749+460A. The mid-infrared (MIR) light curves in the $w 1$ and $w 2$ bands detected by the Wide-field Infrared Survey Explorer (WISE and NEOWISE-R; Wright et al. 2010; Mainzer et al. 2014) indicate that the brightness in both bands decreases gradually by $\sim 0.3$ mag when the object changes from a Seyfert 1.8 to a Seyfert 1.9 nucleus, consistent with the expectation of the accretion-rate enhancement scenario of the CL phenomenon (e.g., Sheng et al. 2017; Stern et al. 2018; Wang et al. 2019). The possibility of a normal SN mentioned above can be further decreased according to the observed MIR variability with an absolute magnitude in the $w 2$ band of $-23.6 \mathrm{mag}$. This value is marginally larger than the brightest one (i.e., $-22.8 \mathrm{mag}$ at $4.5 \mu \mathrm{m}$ for SN 2010j1) reported by Szalai et al. (2019), who studied a sample of MIR light curves of hundreds of SNe.

With increasing cases of CL-AGNs, a few possible models have recently been proposed (e.g., Wang \& Bon 2021). Based on the statistical studies performed by MacLeod et al. (2019) and Wang et al. $(2019,2020 \mathrm{a}, \mathrm{b})$, there is some evidence for the CL phenomenon being understood by the disk-wind BLR model. In the model, a classical BLR can be sustained if $L / L_{\mathrm{Edd}}$ is above a critical value of $\sim 10^{-6}-10^{-3}$, when the fiducal values of a set of parameters of the disk are adopted (e.g., Nicastro 2000; Elitzur \& Ho 2009). In B3 0749+460A, when the $L / L_{\text {Edd }}$ listed in Table 1 decreases gradually from 0.012 to 0.005 , the classical broad $\mathrm{H} \alpha$ component in fact fades away from 2013 to 2021. This link between the strength of the classical $\mathrm{H} \alpha$ broad component and $L / L_{\mathrm{Edd}}$ can be reinforced if we instead estimate $L_{\text {bol }}$ from the X-ray emission. Our X-ray analysis suggests a decreasing $L / L_{\text {Edd }}$ from 0.017 in 2011 to 0.002 in 2021, when a bolometric correction of $L_{\mathrm{bol}}=16 L_{\mathrm{X}}$ is adopted in the calculation. In addition, it is well known that the double-peaked broad Blamer lines observed frequently in AGNs can originate from gas in a single accretion disk (e.g., Storchi-Bergmann et al. 2017, and references therein). The double-peaked broad $\mathrm{H} \alpha$ component is found to weaken synchronously with the classical broad $\mathrm{H} \alpha$ in the object, implying a disk origin for the classical broad $\mathrm{H} \alpha$ component. Alternatively, Pan et al. (2021) and J. Feng et al. (2021) show that the observed repeat CL-AGNs could be explained by reducing the disk burst period by including the effect of a large-scale magnetic field (e.g., Dexter \& Begelman 2009) in the disk-instability model suggested by Sniegowska et al. (2020).

A transition of the accretion state, similar to that seen in XRBs, has been proposed recently as an explanation of the CL phenomenon (e.g., Ruan et al. 2019; Ai et al. 2020), although it seems that this scenario might not be generally applicable. The CL phenomenon in UGC 3223, a local repeat CL-AGN, is found to be unrelated to the X-ray hardness ratio, but instead related to the X-ray luminosity (Wang et al. 2020b). Following Ai et al. (2020), the hardness ratio $\mathrm{CR}_{\text {soft }} /\left(\mathrm{CR}_{\text {soft }}+\mathrm{CR}_{\text {hard }}\right)$ is plotted against $L / L_{\mathrm{Edd}}$ in Figure 7 for B3 0749+460A, where $L / L_{\mathrm{Edd}}$ is derived from the unabsorbed X-ray 2-10 keV luminosity by assuming a bolometric correction of $L_{\mathrm{bol}}=16 L_{\mathrm{X}}$. After converting the photon index $\Gamma$ to the hardness ratio, the $\Gamma-L / L_{\text {Edd }}$ relationships for low-luminosity AGNs (Constantin et al. 2009) and bright AGNs (Risaliti et al. 2009) are overplotted in the figure, which stand for an advection-dominated accretion flow (ADAF) at low $L / L_{\mathrm{Edd}}$ and a standard accretion disk at high $L / L_{\mathrm{Edd}}$, respectively. The $\Gamma-L / L_{\text {Edd }}$ relationships can be well understood by the change of the Compton $y$ parameter with the released energy (e.g., Esin et al. 1997; Janiuk \& Czerny 2000). One can see from the figure that the evolution of the object likely follows the ADAF locus and does not follow the V-shape transitions revealed by $\mathrm{Ai}$ et al. (2020). In fact, assuming the aforementioned disk-wind model, the (dis)appearance of a classical BLR is sensitive to accretion (wind) luminosity in individual AGNs, suggesting that a change of accretion mode is possibly a necessary (rather than a sufficient) condition for the CL phenomenon.

Acknowledgements The authors thank the anonymous referee for a careful review and helpful suggestions that greatly improved the manuscript. This study is supported by the National Natural Science Foundation of China under grant 11773036, and by the Strategic Pioneer Program on Space Science, Chinese Academy of Sciences, grants XDA15052600 and XDA15016500. The authors are grateful for support from the National Key Research and Development Project of China (grant 2020YFE0202100). J.W. is supported by the Natural Science Foundation of Guangxi (2020GXNSFDA238018) and by the 


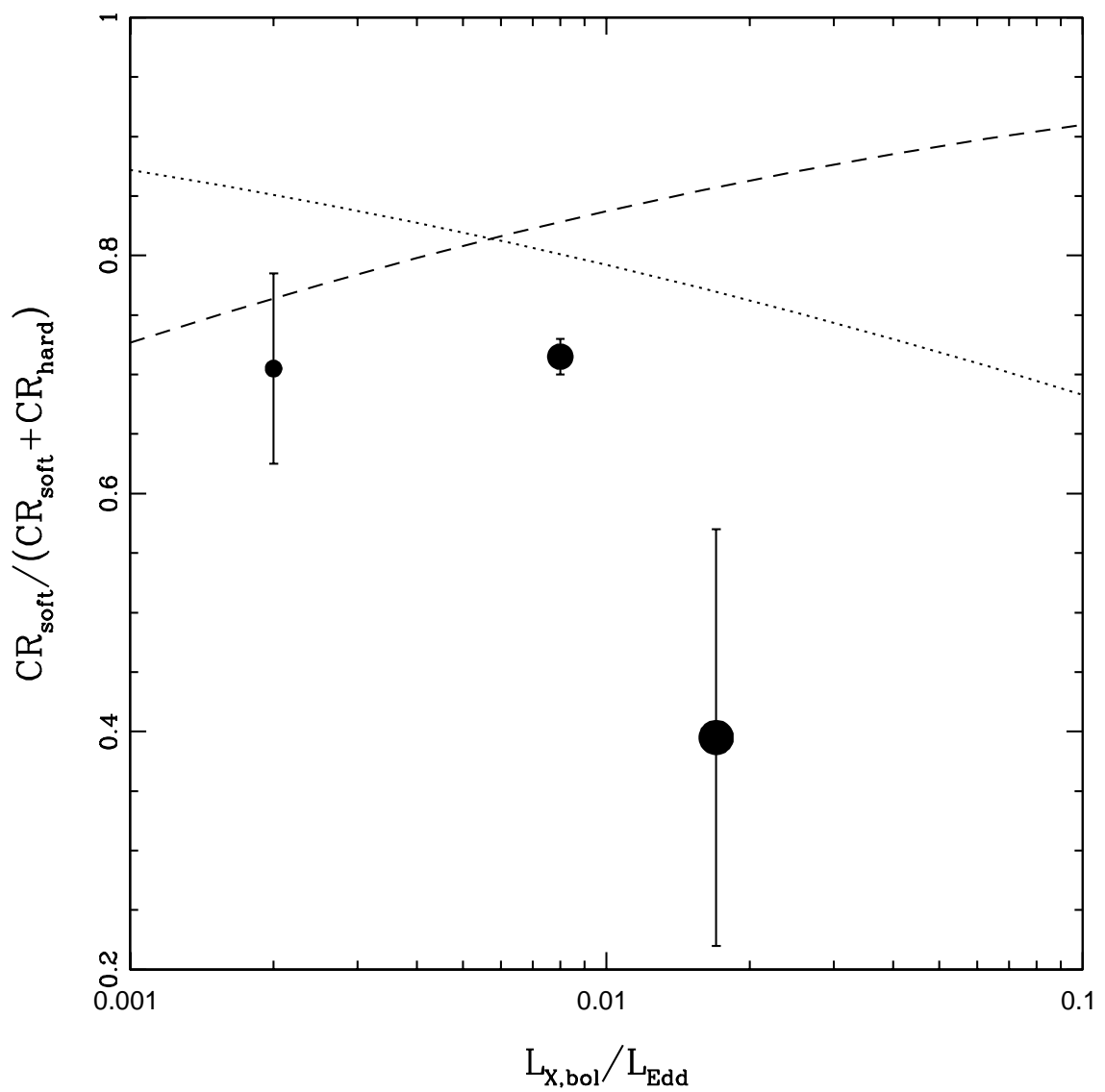

Fig. 7 Hardness ratio plotted against the $L / L_{\mathrm{Edd}}$ for B3 0749+460A. $\mathrm{CR}_{\text {soft }}$ and $\mathrm{CR}_{\text {hard }}$ denote the count rates at $0.2-2$ and $2-12 \mathrm{keV}$, respectively. $L / L_{\text {Edd }}$ is estimated from the unabsorbed $2-10 \mathrm{keV}$ luminosity after assuming a bolometric correction of $L_{\mathrm{bol}}=16 L_{\mathrm{X}}$. The symbol size is proportional to the broad $\mathrm{H} \alpha$ emission. The $\Gamma-L / L_{\mathrm{Edd}}$ relationships of low-luminosity AGNs and bright AGNs are denoted by the dotted and dashed lines, respectively.

Bagui Young Scholars Program. This study is supported by the Open Project Program of the Key Laboratory of Optical Astronomy, NAOC, CAS. A.V.F.'s group is supported by the Christopher R. Redlich Fund and the Miller Institute for Basic Research in Science (in which A.V.F. is a Senior Miller Fellow). We thank the Swift Acting PI, Brad Cenko, for approving our target-of-opportunity request, as well as the Swift observation team for assistance. This study uses the NASA/IPAC Extragalactic Database (NED), which is operated by the Jet Propulsion Laboratory, California Institute of Technology. It also uses data collected by the Wide-field Infrared Survey Explorer (WISE), which is a joint project of the University of California, Los Angeles, and the Jet Propulsion Laboratory/California Institute of Technology, funded by the National Aeronautics and Space Administration (NASA).

\section{References}

Ai, Y. L., Dou, L. M., Yang, C. W., et al. 2020, ApJ, 890, 29

Antonucci, R. R. J. 1993, ARA\&A, 31, 473

Arnaud, K. A. 1996, ASPC, 101, 17 
Bon, E., Gavrilovic, N., La Mura, G., \& Popovic, L. C. 2009a, NewAR, 53, 121

Bon, N., Bon, E., Marziani, P., \& Jovanovic, P. 2015, Ap\&SS,360, 41

Bon, E., Popovic, L. C., Gavrilovic, N., La Mura, G., \& Mediavilla, E. 2009b, MNRAS, 400, 924

Boroson, T. A. 2005, AJ, 130, 381

Bruzual, G., \& Charlot, S. 2003, MNRAS, 344, 1000

Cardelli, J. A., Clayton, G. C., \& Mathis, J. S. 1989, ApJ, 345, 245

Cash, W. 1979, ApJ, 228, 939

Chen, K., Halpern, J. P., \& Filippenko, A. V. 1989, ApJ, 339, 742

Constantin, A., Green, P., Aldcroft, T., Kim, D. -W, Haggard, D, Barkhouse, W., \& Anderson, S. F. 2009, ApJ, 705, 1336

Dexter, J., \& Begelman, M. C. 2019, MNRAS, 483, L17

Drake, A. J., Djorgovski, S. G., Mahabal, A., et al. 2011, in New Horizons in Time-Domain Astronomy, ed. E. Griffin, R. J. Hanisch, \& R. L. Seaman (Proceedings of the International Astronomical Union, Vol. 7, No. S285), 306.

Elitzur, M., \& Ho, L. C. 2009, ApJ, 701, 91

Elitzur, M., \& Shlosman, I. 2006, ApJ, 648, 101

Eracleous, M., \& Halpern, J. P. 1994, ApJS, 90, 1

Esin, A. A., McClintock, J. E., \& Narayan, R. 1997, ApJ, 489, 865

Evans, P. A., Page, K. L., Osborne, J. P., et al. 2020, ApJS, 247, 54

Fan, Z., Wang, H., Jiang, X., et al. 2016, PASP, 128, 115005

Feng, H.-C., Hu, C., Li, S.-S., et al. 2021, ApJ, 909, 18

Feng, J. J., Cao, X. W., Li, J. W., \& Gu, W. M., 2021, ApJ, 961, 61

Filippenko, A. V. 1982, PASP, 94, 715

Frederick, S., Gezari, S., Graham, M. J., et al. 2019, ApJ, 883, 31

Gehrels, N., Chincarini, G., Giommi, P., et al. 2004, ApJ, 611, 1005

Gezari, S., Hung, T., Cenko, S. B., et al. 2017, ApJ, 835, 144

Graham, M. J., Ross, N. P., Stern, D., et al. 2020, MNRAS, 491, 4925

Greene, J. E., \& Ho, L. C. 2005, ApJ, 630,122

Greene, J. E., \& Ho, L. C. 2007, ApJ, 670, 92

Grimm, H. -J., Gilfanov, M., \& Sunyaev, R. 2003, MNRAS, 339, 793

Guo, H. X., Sun, M. Y., Liu, X., Wang, T. G., Kong, M. Z., Wang, S., Sheng, Z. F., \& He, Z. C. 2019, ApJ, 833, 44

Halpern, J. P., \& Filippenko, A. V. 1988, Nature, 331, 46

Hao, C.-N., Kennicutt, R. C., Johnson, B. D., Calzetti, D., Dale, D. A., \& Moustakas, J. 2011, ApJ, 741, 124

Harrison, C. M., Alexander, D. M., Mullaney, J. R., \& Swinbank, A. M. 2014, MNRAS, 441, 3306

Hon, W. J., Webster, R., \& Wolf, C. 2020, MNRAS, 497, 192

Huffman, D. R. 1977, AdPhy, 26, 129

Humphrey, P. J., Liu, W. H., \& Buote, D. A. 2009, ApJ, 693, 822

Hutsemekers D., Agis Gonzalez B., Marin F., Sluse D., Ramos Almeida C., Acosta Pulido J.-A., 2019, A\&A, 625, A54

Janiuk, A., \& Czerny, B. 2000, NewA, 5, 7

Kaastra, J. S. 2017, A\&A, 605, 51

Kalberla, P. M. W., Burton, W. B., Hartmann, D., Arnal, E. M., Bajaja, E., Morras, R., \& Poppel, W. G. L. 2005, A\&A, 440, 775

Kaspi, S., Smith, P. S., Netzer, H., et al. 2000, ApJ, 533, 631

Kollatschny, W., Grupe, D., Parker, M. L., et al. 2020, A\&A, 638, 91

Kollatschny, W., Ochmann, M. W., Zetzl, M., Haas, M., Chelouche, D., Kaspi, S., Pozo Nuñez, F., \& Grupe, D. 2018, A\&A, 619, 168

Kriss, G. 1994, in ASP Conf. Ser. 61, Astronomical Data Analysis Software and Systems III, ed. D. R. Crabtree, R. J. Hanisch, \& J. Barnes (San Fransisco, CA: ASP), 437

LaMassa, S. M., Cales, S., Moran, E. C., et al. 2015, ApJ, 800, 144 
Law, N. M., Kulkarni, S. R., Dekany, R. G., et al. 2009, PASP, 121, 1395

Lawrence, A. 2018, Nature Astronomy, 2, 102

MacLeod, C. L., Green, P. J., Anderson, S. F., et al. 2019, ApJ, 874, 8

MacLeod, C. L., Ivezic, Z., Kochanek, C. S., et al. 2010, ApJ, 721, 1014

MacLeod, C. L., Ross, N. P., Lawrence, A., et al. 2016, MNRAS, 457, 389

Mainzer, A., Bauer, J., Cutri, R. M., et al. 2014, ApJ, 792, 30

Marin, F., Hutsemekers, D., \& Agis Gonzalez, B. 2019, in Proceedings of the 2019 Annual Conference of the SF2A, arXiv:1909.02801

Massey, P., Strobel, K., Barnes, J. V., et al. 1988, ApJ, 328, 315

McElroy, R. E., Husemann, B., Croom, S. M., et al. 2016, A\&A, 593, L8

Miller, J. S., \& Stone, R. P. S. 1993, Lick Obs. Tech. Rep. 66 (Santa Cruz, CA: Lick Observatory)

Nicastro, F. 2000, ApJ, 530, 65

Osterbrock, D. E., \& Ferland, G. J. 2006, Astrophysics of Gaseous Nebulae and Active Galactic Nuclei (2nd ed.; Sausalito, CA: University Science Books)

Pan, X., Li, S. -L., \& Cao, X. 2021, arXiv:astro-ph/2103:00828, accetped by ApJ

Parker, M. L., Komossa, S., Kollatschny, W., et al. 2016, MNRAS, 461, 1927

Peterson, B. M., McHardy, I. M., Wilkes, B. J., et al. 2000, ApJ, 542, 161

Popovic, L. C., Mediavilla, E., Bon, E., \& Ilic, D. 2004, A\&A, 423, 909

Popovic, L. C., Mediavilla, E. G., Bon, E., Stanic, N., \& Kubicela, A. 2003, ApJ, 599, 185

Risaliti, G., Young, M., \& Elvis, M. 2009, ApJ, 700, 6

Ruan, J. J., Anderson, S. F., Cales, S. L., et al. 2016, ApJ, 826, 188

Ruan, J. J., Anderson, S. F., Eracleous, M., Green, P. J., Haggard, D., MacLeod, C. L. Runnoe, J. C., \& Sobolewska, M. A. 2019, ApJ, 883, 76

Runnoe, J. C., Cales, S., Ruan, J. J., et al. 2016, MNRAS, 455, 1691

Schlafly, E. F., \& Finkbeiner, D. P. 2011, ApJ, 737, 103

Shapovalova, A. I., Popovic, L. C., Burenkov, A. N., et al. 2010, A\&A, 509, 106

Shappee, B. J., Prieto, J. L., Grupe, D., et al. 2014, ApJ, 788, 48

Sheng, Z., Wang, T., Jiang, N., et al. 2017, ApJ, 846, 7

Sheng, Z., Wang, T., Jiang, N., et al. 2020, ApJ, 889, 46

Sniegowska, M., Czerny, B., Bon, E., \& Bon, N. 2020, A\&A, 641, 167

Stern, D., McKernan, B., Graham, M. J., et al. 2018, ApJ, 864, 27

Storchi-Bergmann, T., Schimoia, J. S., Peterson, B. M., Elvis, M., Denney, K. D., Eracleous, M., \& Nemmen, R. S. 2017, ApJ, 835, 236

Strateva, I. V., Strauss, M. A., Hao, L., et al. 2003, AJ, 126, 1720

Szalai, T., Zsiros, S., Fox, O. D., Pejcha, O., \& Muller, T. 2019, ApJS, 241, 38

Tody, D. 1986, Proc. SPIE, 627, 733

Tody, D. 1992, in ASP Conf. Ser. 52, Astronomical Data Analysis Software and Systems II, ed. R. J. Hanisch, R. J. V. Brissenden, \& J. Barnes (San Fransisco, CA: ASP), 173

Trakhtenbrot, B., Arcavi, I., MacLeod, C. L., et al. 2019, ApJ, 883, 94

Veron, P., Goncalves, A. C., \& Veron-Cetty, M. -P. 2002, A\&A, 384, 862

Veron-Cetty, M.-P., \& Veron, P. 2006, A\&A, 455, 773

Wang, J., Mao, Y. F., \& Wei, J. Y. 2011, ApJ, 741, 50

Wang, J., Xu, D. W., Sun, S. S., Feng, Q. C. Li T. R., Xiao, P. F., \& Wei, J. Y. 2020a, AJ, 159, 245

Wang, J., Xu, D. W., Wang, Y., Zhang, J. B., Zheng, J., \& Wei, J. Y. 2019, ApJ, 887, 15

Wang, J., Xu, D. W., \& Wei, J. Y. 2018a, ApJ, 852, 26

Wang, J., Xu, D. W., \& Wei, J. Y. 2018b, ApJ, 858, 49

Wang, J., Xu, D. W., \& Wei, J. Y. 2020b, ApJ, 901, 1

Wang, J. -M., \& Bon, E. 2020, ApJ, 643, 9

Woo, J. H., Son, D., \& Bae, H. J. 2017, ApJ, 839, 120

Wright, E. L., Eisenhardt, P. R. M., Mainzer, A. K., et al. 2010, AJ, 140, 1868

XMM-SSC, 2018, VizieR Online Data Catalog: XMM-Newton slew survey Source Catalogue, version 2.0, https://ui.adsabs.harvard.edu/abs/2018yCat.9053....0X 
Yan, L., Wang, T. G., Jiang, N., et al. 2019, ApJ, 874, 44

Yang, Q., Wu, X. B., Fan, X. H., et al. 2018, ApJ, 862, 109

Zhang, K., Wang, T., Gaskell, C. M., \& Dong, X. 2013, ApJ, 762, 51 